\title{
Students’ Teleologic and Anthropomorphic References to Select Concepts of Environmental Science
}

\author{
Lani Isleta-Palatino* \\ Centre for General Education, AMA International University, Bahrain, Manama, Kingdom of Bahrain \\ *Corresponding author: lipalatino@amaiu.edu.bh \\ Received January 08, 2014; Revised January 16, 2014; Accepted February 18, 2014
}

\begin{abstract}
This study attempted to ascertain the students' teleologic and anthropomorphic references to select concepts of Environmental Science. Using questions forming generalizations on abstract topics of Hydrologic Cycle, Acid Rain, Air Pollution and Global Warming, students' teleologic-anthropomorphic references were determined. It was found out that the students' who had just finished their secondary education program and underwent the English curriculum had the least affordance of teleologic and anthropomorphic references. Statistical analyses, based on the scope of the study, discriminated the following results: classroom pedagogical interventions made a $61.5 \%$ impact on the correction of their teleologic-anthropomorphic references; teleologic and anthropomorphic references were highly significant related to age in an inverse relation and an emerging relationship to the English curriculum was discerned.
\end{abstract}

Keywords: academic learning and cognition, anthropomorphic language, pedagogical interventions, teleologic reference

Cite This Article: Lani Isleta-Palatino, "Students' Teleologic and Anthropomorphic References to Select Concepts of Environmental Science.” American Journal of Educational Research, vol. 2, no. 2 (2014): 102-106. doi: 10.12691/education-2-2-6.

\section{Introduction}

Learning is an active process that takes place in a dynamic environment of sustained community of inquiry. The self-motivated erudition of conceptual cognition to various academic ventures is a result of a shared culture of teaching and learning inside and out of the classroom environment.

Learners construct concepts based on their default knowledge and beliefs which were acquired through mental cognition and reception to various environmental stimuli. The construction of a concept to various phenomena changes as a learner accumulates conceptual knowledge and understanding through cognitive and metacognitve learning strategies.

Anthropomorphism, or the tendency to attribute human motivation, characteristics, or behavior to nonhuman entities, reflects and shapes perception and beliefs to the nonhuman world $[4,11]$. Believed to be attributed to age and exposure to metal cognition and learning, it diminishes as one gets full cognition to a phenomenon through conceptual and scientific explanation through learning experience.

The students' teleologic references and anthropomorphic languages is interpreted in a framework that converts personal beliefs and practices into contextual frameworks affected by their learning exposures through classroom discussions and cognition between teacher and students, student and students, students and select learning modules and other academic infrastructures.
Learning exposure plays a vital role in the re-formation of their capabilities on teleologic references and anthropomorphic reasoning. If not intervened properly by academic learning and cognition, this forms an impetustype of conception which eventually forms mountingroadblocks that can hinder their scientific understanding and explanations to the abstraction process of new concepts.

Teleologic references and anthropomorphic languages among learners had been regarded as an intangible configuration of the mind which in turn plays an active role in the process of constructing their personal knowledge in learning concepts of science.

\subsection{Theories of Anthropomorphism}

\subsubsection{Familiarity Thesis}

The familiarity thesis affirms that we anthropomorphize phenomenon because it allows us to explicate things we do not comprehend in a manner that we understand; hence, what we understand best is ourselves. The familiarity thesis is a primarily cognitive motivation for anthropomorphism: it attempts to understand the world based on a mental model of the world that we are most familiar with [5,7].

\subsubsection{Comfort Thesis}

The comfort thesis is the prime emotional motivation for anthropomorphism. People anthropomorphize because they are not comfortable with things that are not like us; 
hence, "making" things be like us reduces discomfort. According to the comfort thesis, anthropomorphism is "an attempt to feel like we can delineate and persuade the world if it is more like us than not.” [5,7].

\subsubsection{Best-Bet Thesis}

The best-bet thesis is a cognitive and game-theoretic approach to anthropomorphism. The best-bet thesis states that "in a world of chronic uncertainty about the nature of the world, guessing constitutes a good bet on something or to an event that has a human cause: we gain much if we are right and we usually lose little if we are wrong” [5,7].

\subsubsection{Social Thesis}

Caporael and Heyes [3] put forth a theory of anthropomorphism in a term Species-Specific Group-Level Coordination System, which was then translated to social thesis. This thesis claims the psychological discussion of anthropomorphism is not neutral but is in fact value laden and defines our interaction with the environment. "From this perspective, attributing human characteristics to animals is a way of changing the values we place on them and how we can behave towards them". In the social thesis, the act of anthropomorphizing reflects values and possesses the potential for social consequence [7].

\subsubsection{Object Subject Interchangeability}

The concept of object-subject interchangeability proposes that people attribute meaning to other people and objects in the construction, adaptation, and maintenance of the self. The distinction between the influence of other people and objects is not always firm. Anthropomorphism may be used to attribute a human-like quality to an object that has particular salience in defining who we are individually or culturally $[5,8]$.

\subsubsection{Phenomenological Inter-subjectivity}

The concept of phenomenological inter-subjectivity proposes that anthropomorphism is a reflection of how we experience and order the world. This concept argues we experience objects that seem to be animated by human consciousness and will, causing the distinction between self and other to be blurred, and that anthropomorphism is a pragmatic response to such objects in order to make sense of them $[5,8]$.

\subsection{Van Fraassen (VF) Pragmatic Theory of Explanation}

VF theory of explanation is essentially a theory of whyquestions. According to Van Fraassen, formulating a theory of explanation poses two main problems: (1) the provision of an account of justifiable rejection of explanation-request, and (2) the accounting for the asymmetries of explanation. To this end, he takes explanation as a ternary relationship that holds between theories, facts and contexts.

Why-questions and their answers are individuated only relative to a context. The point of construing a contextual analysis of explanation is to put the concept of explanation on grounds that are respectable from an empiricist standpoint. VF takes the "received view" as characterizing explanation as a relationship between theory and fact. This cognition ends up to the existence of causal relationships beyond the observable phenomena [1].

\subsection{Objectives of the Study}

This study attempted to analyze the students' teleologic and anthropomorphic references to select concepts of Environmental Science.

Specifically, it sought to find explanations of the following:

1. What is the extent of students' preconceptions on select concepts in Environmental Science as a result of their affordance of teleologic-anthropomorphic references?

2. Do classroom pedagogical interventions correct the students' teleologic-anthropomorphic references on select concepts in Environmental Science?

3. Which learning factor is associated with the students' teleologic-anthropomorphic reference on select concepts in Environmental Science?

\section{Methodology}

This study made use of Descriptive-Correlation method in ascertaining the preconceived research problems. It involved a systematic investigation using pre-assessment results, formative evaluations, interview and questionnaire as predominant methods of data collection. Corroboration of findings, vis-à-vis with the identified norms of the context of the study, was used to conclude on the students' teleologic and anthropomorphic references on select concepts of Environmental Science.

The respondents of the study were the 40 students of the author in Environmental Science at AMA International University in the Kingdom of Bahrain for the first trimester, AY 2012.

Pearson-r correlation was used in determining the relationship of select variables to their teleologic and anthropomorphic references while ANCOVA results were utilized to determine which of the variables used is cogently discriminated in the process.

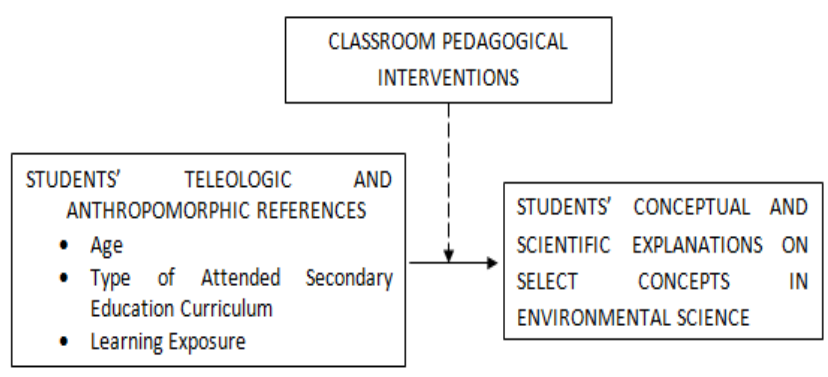

Figure 1. Research Paradigm

Presented in the foregoing figure is the paradigm of the study. It made use of the assumptions that teleologic explanation and anthropomorphic language of the students are influenced by their age and learning exposure. Moreover, it also assumes that their learning exposure is dependent on the type of their attended secondary education curriculum. In this set up, there are two curriculums which the respondents are grouped of: English and Arabic. The English curriculum was used by the International and Private Schools and the Philippine School-Bahrain while the Arabic Curriculum was the 
public Science Curriculum. These variables are believed to be the respondents' input on their affordance of teleologic explanation and anthropomorphic language on select concepts in Environmental Science.

On the other hand, the teleologic explanation and anthropomorphic language of the respondents are expected to be corrected by classroom pedagogical interventions. The interventions made were multimedia presentations, classroom interactions and other class works based on notional hours to complement the designed learning competencies to hone their background understanding of the subject. These cognitions help them formulate new concepts and scientific explanations on select concepts in Environmental Science: Hydrologic Cycle, Acid Rain, Air Pollution and Global Warming.

Table 1. The Extent of Preconceptions of the Students on Select Concepts in Environmental Science as a Result of their TeleologicAnthropomorphic References

\begin{tabular}{|c|c|c|c|c|c|c|}
\hline Age & $\mathrm{F}$ & D.I. & Topics & D.I. & $\mathrm{F}$ & Curriculum \\
\hline $16-17$ & 1.15 & $\mathrm{~L}$ & \multirow{3}{*}{ Hydrologic Cycle } & $\mathrm{L}$ & 1.24 & English \\
\hline $18-19$ & 1.63 & M & & M & 1.79 & Arabic \\
\hline 20 and above & 2.29 & M & & & & \\
\hline $16-17$ & 1.45 & $\mathrm{~L}$ & \multirow{3}{*}{ Acid Rain } & $\mathrm{M}$ & 1.62 & English \\
\hline 18-19 & 2.00 & M & & M & 1.93 & Arabic \\
\hline 20 and above & 2.57 & $\mathrm{H}$ & & & & \\
\hline $16-17$ & 1.42 & $\mathrm{~L}$ & \multirow{2}{*}{ Air Pollution } & M & 1.53 & English \\
\hline 20 and above & 2.00 & $\mathrm{H}$ & & & & \\
\hline $16-17$ & 1.00 & $\mathrm{~L}$ & \multirow{3}{*}{ Global Warming } & $\mathrm{L}$ & 1.06 & English \\
\hline 18-19 & 1.13 & $\mathrm{~L}$ & & $\mathrm{~L}$ & 1.36 & Arabic \\
\hline 20 and above & 1.86 & $\mathrm{M}$ & & & & \\
\hline
\end{tabular}

Legend: F - Frequency; DI - Descriptive Interpretation: L - Low; M - Moderate; H - High

Presented in the foregoing table are the extents of preconceptions of the student-respondents as a result of their teleologic-anthropomorphic references in eliciting concepts in Environmental Science when grouped according to age and type of secondary education attended.

Using questions forming generalizations on select topics of Hydrologic Cycle, Acid Rain, Air Pollution and Global Warming, students' teleologic-anthropomorphic reference was determined. It can be said that the students' who had just completed their secondary education had the least affordance of teleologic-anthropomorphic reference when compared to their counterparts.

Concomitantly, the students who completed English curriculum in their Secondary Science Program had the least affordance of teleologic-anthropomorphic references when compared to their counterparts who had the Arabic curriculum. This phenomenon can be attributed to the fact that the medium of instruction used in the present study is
English. This attribution is believed to be useful as students who have both the linguistic and semantic competences in English can easily infer on phenomenon and validate generalizations based on facts [2].

The foregoing results can be construed by the conjectures stipulated in Piaget's Cognitive Development Theory which in turn used by the students in their construction of pragmatic explanations to various phenomenon (VF Theory of Explanation) [1]. It was posited that language takes part in the cognitive development of a person. Concomitantly, their linguistic and semantic competences discriminates their concept abstraction abilities in forming a functional schema to a ready and available conceptual understanding. This functional schema takes a potent role in resembling a pragmatic explanation in the verbal mode of the student in explaining abstract concepts [1,2].

Table 2. Tests of Between-Subjects Effects of Classroom Pedagogical Interventions on the Students' Affordance of Teleologic and Anthropomorphic Reference in relation to their Age and Curricular Exposure

\begin{tabular}{|c|c|c|c|c|c|}
\hline Source & Type III Sum of Squares & df & Mean Square & $\mathrm{F}$ & Sig. \\
\hline Corrected Model & $9.707^{\mathrm{a}}$ & 6 & 1.618 & 13.501 & .000 \\
\hline Teleologic and Anthropomorphic Reference & 8.693 & 1 & 8.693 & 72.547 & .000 \\
\hline Age & 1.615 & 2 & .807 & 6.737 & .003 \\
\hline Curriculum & .798 & 1 & .798 & 6.663 & .014 \\
\hline Age $*$ Curriculum & .852 & 2 & .426 & 3.554 & .038 \\
\hline
\end{tabular}

a. R Squared =. 664 (Adjusted R Squared =. 615)

Presented in the table are the tests of between-subjects effects of classroom pedagogical interventions on the students' affordance of teleologic and anthropomorphic references on select concepts in Environmental Science.

It was found out that there is a significant difference on the students' affordance of teleologic and anthropomorphic reference after various classroom pedagogical interventions had been introduced: F-value of 13.501 and a p-value of $<.001$, at. 05 level of significance. Hence, it can be said that teleologic and anthropomorphic references can be corrected as student-learners are confronted with various classroom pedagogical interventions.

The interactions of the classroom pedagogical interventions to the respondents' affordance of teleologic and anthropomorphic references were also analyzed when the respondents were grouped according to their age and curricular undertakings: F-values of 6.737 and 6.663, and p-values of 0.003 and 0.014 , at. 05 level of significance, respectively. Hence, it can be said that anthropomorphism reduces as one gets expose to conceptual explanation through curricular learning experiences.

It may be noted, however, that the impact of the classroom pedagogical interventions is moderately high considering that the coefficient of determination indicated by the adjusted R-squared is $61.5 \%$. This means that the pedagogical interventions done in the subject account for $61.5 \%$ of the variability in the teleologic and anthropomorphic reasoning of the students. It is construed 
then that there are other important variables or factors that affect the students' teleologic and anthropomorphic reference, e.g., cognitive and metacognitive abilities, attitude and motivation in learning Physics.

Table 2 likewise presents the interaction between classroom pedagogical interventions and the age and curricular undertakings of student-respondents. It presents the impact of the interventions done in the study: F-value of 3.554 and p-value of. 038 at. 05 level of significance. These results were analyzed using the estimated marginal means with a covariate value of 1.45 as shown in Figure 2.

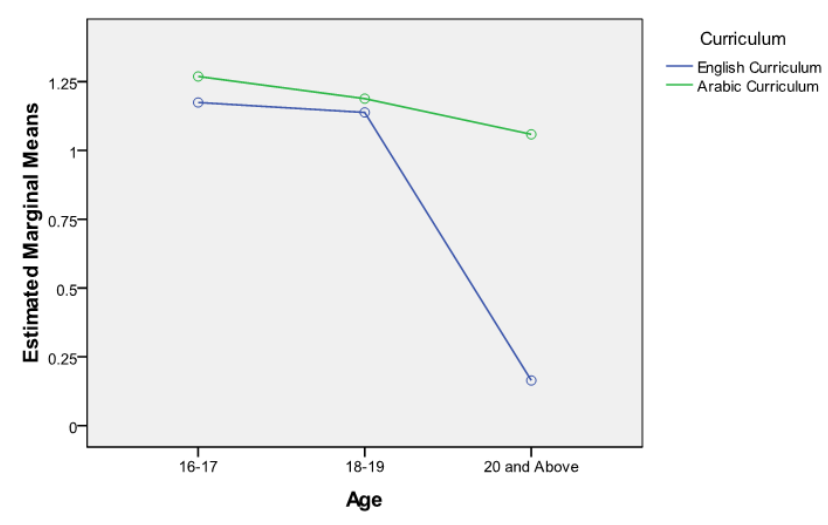

Covariates appearing in the model are evaluated at the following values: Initial $=1.45$

Figure 2. Estimated Marginal Means of Between-Subjects Effects of Classroom Pedagogical Interventions on the Students' Teleologic and Anthropomorphic References in relation to their Age and Curricular Exposure

Figure 2 presents the relationship of the estimated marginal means of the post-affordance results and the age of the respondents' concomitant to their curricular undertakings. The result of the post-test mean score is evaluated with the pre-affordance covariate value of 1.45 . It presents that the students who were exposed to the English curriculum had the least affordance when compared to their Arabic counterparts across age brackets. Concomitantly, the interaction is highly evident to the affordance of the respondents at the age bracket of 20 and above.

These findings support claims of various researchers when they said that educational procedures and learning exposures alleviate the learner's tendency to anthropomorphize [9]. Moreover, it was concluded that anthropomorphic utterances are formed subsets of students' alternative responses brought about by their intuitions and metacognitions. Hence, students use their prior knowledge in a range of self-reflexivity in their abilities to anthropomorphize [2,6].

Hence, the typology and heuristics of conceptual thinking among students must also be considered. It is said that the human mind has evolved heuristics by using its prior experiences in bridging the missing, lost and damaged in a framework of conceptual thinking and understanding. It is in this context that the human mind conceptualizes and re-conceptualizes concepts based on his experiences and available explanations within his schema [10].

On the other hand, the theories of anthropomorphism explicate well the transition of the different levels of understanding that leads a person formulate his central schema of thinking and reasoning. Corollary to Piaget's
Cognitive Development Theory, these beliefs, which are innate to the person, become his central schema in formulating a pragmatic explanation to a certain phenomenon. The theory explicates further the nature of a person's conceptual knowledge and understanding and how he comes to gain, create and apply it to various mental exercise and activities. Cognitive development, in this sense, is a progressive restructuring of intellectual progressions as a result of the learner's biological maturation and environmental experiences. Learners construct a conceptual understanding on phenomena that takes place around him through his experiences and to what he already knows. They also tend to discover understanding through his environment. Hence, the theory conjectures the idea that cognitive development happens at the center of human organism and language is dependent to cognitive development $[3,5,7,8]$.

This phenomenon can be attributed to the skill and will under the self-regulation of the student-learner. This supports the fact that 'A cognitive learning strategy is a plan for orchestrating cognitive resources, such as attention and long-term memory to help reach a learning goal'. Astutely, it can be concluded that the learning abilities of the student-learners and competencies of recalling past knowledge in formulating concepts is controlled by their cognitive and metacognitive strategies. The determinant and selection of the cognitive and metacognitive learning strategies is determined by their ability and mastery in English. Thus, students develop a greater understanding of themselves as language learners. They become more actively and personally involved in the learning process, more confident, more curious and ask more questions, and develop strong motivation and positive attitudes towards learning [2].

Therefore, it can be said that teleologic and anthropomorphic references to elicit concepts in Environmental Science forms a dichotomy of understanding: a springboard to develop a scientific explanation as one is exposed to further studies and explanation. It was hypothesized that anthropomorphism is just a stage in the development of an understanding [12] This affordance is expected to diminish as other levels of explanation become available (cognitive and metacognitive understanding) [2,12].

Table 3. The Attribution of the Students' Teleologic and Anthropomorphic Reference to their Age and Curricular Undertakings

\begin{tabular}{ccc}
\hline & \multicolumn{2}{c}{ Teleologic and Anthropomorphic Reference of the } \\
& \multicolumn{2}{c}{ Respondents } \\
\hline \multirow{2}{*}{ Age } & Pearson Correlation & $-.470^{* *}$ \\
& Sig. (2-tailed) & .001 \\
\multirow{2}{*}{ Curriculum } & Pearson Correlation & .208 \\
& Sig. (2-tailed) & .156 \\
\hline
\end{tabular}

**. Correlation is significant at the 0.01 level (2-tailed).

Presented in the foregoing table is the attribution of the students' teleologic and anthropomorphic reference to their age and curricular undertakings.

As can be gleaned on the table, the teleologic explanations and anthropomorphic reference of the student-respondents are attributed to their age: r-value of .470 and p-value of. 001, at. 01 level of significance. This means that their teleologic explanations and anthropomorphic languages diminish as they ages. It can be construed further that learning corrects their 
misconceptions to a certain phenomenon. These results confirm the assumption of the study which says that teleologic explanations and anthropomorphic languages of students are related to their age.

On the other hand, curriculum is not significantly related to their teleologic explanations and anthropomorphic languages: r-value of. 208 and p-value of. 156, at. 05 level of significance. However, an emerging positive correlation is getting evident as indicated by the results value.

\section{Conclusion}

Based on the findings of the study, the following are concluded: (1) the students who completed English curriculum in their Secondary Science Program had the least affordance of teleologic-anthropomorphic references when compared to their counterparts who had the Arabic curriculum; (2) students' teleologic-anthropomorphic references can be corrected by classroom pedagogical interventions; and (3) teleologic and anthropomorphic references is highly significant related to age in an inverse relation and an emerging relationship to the English curriculum was discerned.

\section{Recommendation}

Based on the conclusions of the study, the following are forwarded: (1) the use of English, as medium of instruction, is highly recommended to equip students comparable learning experiences with their counterparts, e.g. local, regional, national and international. This phenomenon is necessary in creating a bar-none curriculum which is fundamental in rationalizing the world's educational system - the internalization in higher education; (2) Teachers, as facilitator of student learning, should exude extra effort in the teaching-learning process as they play a critical role in reaping and reducing the drawbacks of the contrasting learning modes of studentlearner. In this manner, students' teleologicanthropomorphic references maybe corrected; (3) divergent learning experiences, in all learning platforms, are highly recommended to harness students' potentials in an integrated learning mechanism.

\section{References}

[1] Bautista, R.G. The students' procedural fluency and writtenmathematical explanation on constructed response tasks in physics. Journal of Technology and Science Education (JOTSE), 3(1), 2013, 49-56.

[2] Bautista, R.G. The Impact of Cognitive and Metacognitive Learning Strategies in Desktop Teaching. Anglisticum international Journal of Literature, Linguistics and Interdisciplinary Studies. Vol. 1, N0.2, 135-143, 2012.

[3] Caporael, L.R., and Heyes, C.M. Why Anthropomorphize? Folk Psychology and Other Stories, in Anthropomorphism, Anecdotes, and Animals (Mitchell, R.W, Thompson, N.S, Miles, H.L eds.). State University of New York Press, Albany, New York, 1997, 59-73.

[4] Chartrand, T. L., et al. Automatic Effects of Anthropomorphized Objects on Behavior. Social Cognition. Vol 26, No 2, 198-209, 2008.

[5] DiSalvo, C. and Gemperle, F. From Seduction to Fulfillment: The Use of Anthropomorphic Form in Design. State University of New York Press, Albany, New York. 2003.

[6] Dorion, K. A Learner's Tactic: How Secondary Students' Anthropomorphic Language may Support Learning of Abstract Science Concepts. Electronic Journal of Science Education. Vol. 12, No. 2. 2011 Retrieved from http://ejse.southwestern.edu.

[7] Guthrie, S.E. Anthropomorphism: A Definition and a Theory in Anthropomorphism, Anecdotes, and Animals (Mitchell, R.W, Thompson, N.S, Miles, H.L eds.). State University of New York Press, Albany, New York. 1997, 50-58.

[8] Jackson, M. Familiar and Foreign Bodies, A Phenomenological Exploration of the Human-Technology Interface. Journal of the Royal Anthropology Institute, Vol. 8, 333-346, 2002.

[9] Kelemen, D., and Rosset, E. The Human Function Compunction: Teleological Explanation in Adults. Social Cognition, 138-143, 2009.

[10] Leboe, J and Whittlesea, B. The Inferential Basis of Familiarity and Recall: Evidence for a Common Underlying Process. Journal of Memory and Language, 804-829, 2002.

[11] Pickett, J. P., et al.. Anthropomorphism. The American Heritage Dictionary of the English Language, (4th Ed.). Boston: Houghton Mifflin Company. 2000.

[12] Taber, K.S. and Watts, M. The Secret Life of the Chemical Bond: Students' Anthropomorphic and Animistic References to Bonding. International Journal of Science Education, Vol 18, No. 5. 123158, 1996. 